\section{Acute Pectoralis Major Tears in Forward Deployed Active Duty U.S. Military Personnel: A Population at Risk?}

\begin{abstract}
Introduction: As sport related activities and weight training broaden in popularity, the incidence of pectoralis major tears appears to be increasing. The purpose of our study is to report on the causes of a series of acute pectoralis major tears in active duty deployed military personnel; identify any patient specific risk factors and to confirm that in-theater surgical repair is possible in most cases.

Methods: Retrospective analysis of the medical records and imaging of 9 cases of acute pectoralis major tears evaluated and treated by the deployed military orthopedic surgeons at one expeditionary medical treatment facility (MTF) over a 4 month deployment cycle from December 2013 through March 2014 was performed.

Results: Nine male patients (5 USAF, USA 4) were diagnosed with pectoralis major tears; 7 complete tears at the tendinous insertion (Tietjen Type III-D), 1 complete tear at the musculotendinous junction (Tietjen Type III-C) and 1 incomplete tear (Tietjen Type II) with a mean age of 32 years (23-52). All injuries occurred during bench press with a mean weight of $258 \mathrm{lbs}$ (135-415 lbs), with the dominant upper extremity involved $56 \%$ of the time. Mean length of deployment was 230 days (120-365), with injury occurring an average of 77 days into the tour (3-198 days). Mean time from injury to surgical repair in the cohort was 18 days $(10-43)$.

Conclusion: Due to the alarming frequency with which pectoralis major ruptures were diagnosed and treated at one expeditionary military treatment facility over a short four-month deployment cycle; combat deployed active duty US military personnel likely represen a high-risk population for this injury. Surgical repair with a trough and transosseous repair technique is possible in the forward deployed setting and may be considered on a case-by-case basis.
\end{abstract}

\section{Introduction}

Rupture of the pectoralis major has been regarded as an uncommon injury, however recent literature has demonstrated a substantial increase in the number of reported cases [1-4]. The over whelming majority of cases have occurred in the last twenty years and the frequency continues to climb $[1,2,4,5]$. Indirect injuries associated with weight lifting are increasingly more common, coinciding with the rise in bench press injuries [5]. During the deep part of the bench press repetition, the pectoralis major tendon is most prone to rupture during eccentric contraction with the arm positioned in 30 degrees of extension and 40 degrees of abduction [6]. In this position the inferior most fibers of the tendon are at a distinct mechanical disadvantage and undergo twice the excursion of the superior fibers [6]. As sport related activities and weight training broaden in popularity, the incidence of pectoralis major tears appears to increase $[1,2,5,7]$.

Due to the rarity of this condition, it may be missed on initial presentation or misdiagnosed as a sprain leading to delay in proper diagnosis and subsequent treatment. Recent articles in the medical literature are now focused on the diagnosis, management, surgical options, and identification of independent risk factors. Although case

\section{Journal of}

Orthopedics \& Rheumatology

\section{Dane Salazar ${ }^{1,2 *}$, Irshad Shakir ${ }^{1}$, Heidi Israel ${ }^{1}$, W. Steven Choate ${ }^{3}$, Keith Joe $^{4}$ and Kara Van de Kieft $^{5}$}

${ }^{\text {I} D i v i s i o n ~ o f ~ O r t h o p a e d i c ~ T r a u m a, ~ D e p a r t m e n t ~ o f ~ O r t h o p a e d i c ~}$ Surgery, Saint Louis University School of Medicine, USA

${ }^{2}$ United States Air Force Center for Sustainment of Trauma and Readiness Skills (C-STARS), University of Maryland, USA

${ }^{3}$ Department of Orthopaedic Surgery, 56th Medical Group, Luke Air Force Base, Arizona, USA

${ }^{4}$ Department of Orthopedic Surgery, 779th Medical Group, Andrews Air Force Base, USA

${ }^{5}$ Department of Radiology, 59th Medical Wing, Wilford Hall Ambulatory Surgical Center, Lackland Air Force Base, USA

\section{*Address for Correspondence}

Dane Salazar MD, Division of Orthopaedic Trauma, Department of Orthopaedic Surgery, Saint Louis University School of Medicine, Saint Louis, MO 63104, USA, Tel: 314-577-8850; Fax: 314-268-5121; Email: dsalazar@lumc.edu

Submission: 03 February, 2018

Accepted: 05 March, 2018

Published: 13 March, 2018

Copyright: () 2018 Salazar D, et al. This is an open access article distributed under the Creative Commons Attribution License, which permits unrestricted use, distribution, and reproduction in any medium, provided the original work is properly cited.

reports and a small three patient case series of pectoralis major repairs in active duty U.S. military personnel have been previously reported, there has never been an investigation of pectoralis major tears in forward deployed active duty troops [8-10]. The purpose of our study is to report on the causes of a series of pectoralis major tears at one expeditionary medical treatment facility (MTF) over a four month deployment cycle, identify any patient specific risk factors and to confirm that in-theater surgical repair is possible in most cases.

\section{Methods}

After institutional review board approval, retrospective analysis of the medical records and imaging of 8 cases of pectoralis major tears evaluated and treated by the deployed United States Air Force

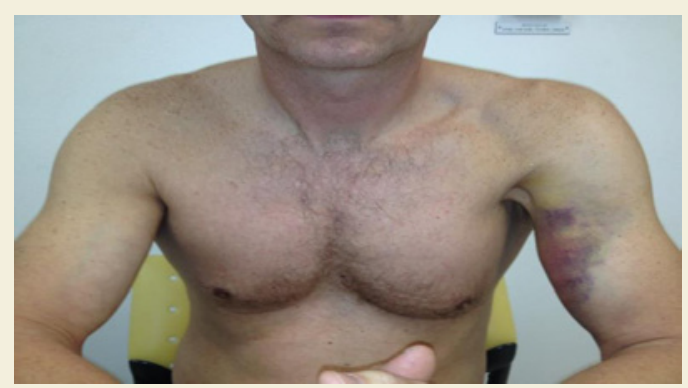

Figure 1: Clinical picture of left pectoralis major tear from our military cohort. Note the loss of the anterior axillary fold and associated ecchymosis on the left. 
Citation: Salazar D, Shakir I, Israel H, Choate WS, Joe K, et al. Acute Pectoralis Major Tears in Forward Deployed Active Duty U.S. Military Personnel: A Population at Risk?. J Orthopedics Rheumatol. 2018; 5(1): 6.

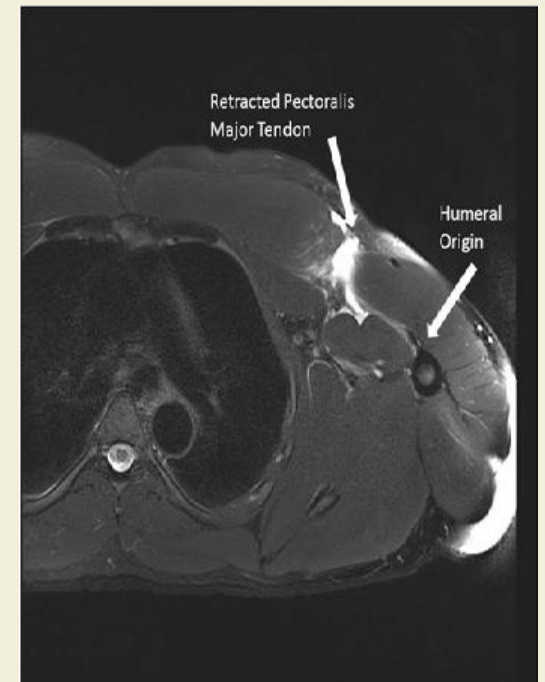

Figure 2: Axial T2 MRI images showing pectoralis major avulsion off the humeral insertion.

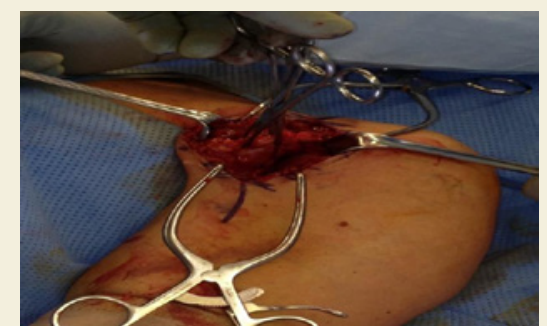

Figure 3: Identification and securing of the retracted tendon stump.

Table 1: Tietjen's classification of pectoralis major injuries.

\begin{tabular}{|l|l|}
\hline \multicolumn{1}{|c|}{ Type } & \multicolumn{1}{c|}{ Injury Pattern } \\
\hline I & Contusion or Sprain \\
\hline II & Partial Tear \\
\hline III & Complete Tear \\
\hline III-A & Muscle Origin \\
\hline III-B & Muscle Belly \\
\hline III-C & Musculotendinous Junction \\
\hline III-D & Tendinous Insertion \\
\hline
\end{tabular}

orthopedic surgeons at one expeditionary medical treatment facility (MTF) over a 4 month deployment cycle from December 2013 through March 2014 was performed. For purposes of operational security the exact location of the MTF will remain undisclosed. All eight subjects were evaluated and treated by one of the facilities' two orthopedic surgeons (DS and WSC). Preoperative radiographs were obtained on each subject, and orthogonal shoulder views were used to rule out avulsion fractures. Diagnosis was made based on history and physical examination (Figure 1). Additionally for 6 of the 8 patients MRI scans were performed at surrounding local foreign hospitals and available for review to confirm the diagnosis and aid in preoperative planning (Figure 2). Patient demographic data, including age, gender, rank, hand dominance, mechanism of injury, history of tobacco usage, and preexisting medical conditions, were recorded and used for comparisons. The Tietjen classification system of pectoralis major injuries was used to record the location and severity of each patient's rupture (Table 1) [11].

\section{Surgery}

All surgeries were performed under general anesthesia, and local interscalene blocks were used to aid with intraoperative and postoperative pain control. The distal portion of a standard deltopectoral incision was utilized for the approach. The cephalic vein was identified and taken laterally. Using palpation and blunt dissection the medially retracted stump of the pectoralis major tendon was identified and secured. Two \#2 ultra strong Kevlar core sutures (Arthrex, Naples FL) were then placed in a locking Krachow fashion into the stump of the pectoralis major tendon (Figure 3). The long head of the biceps was then identified in the bicipital groove. In 5 of the 6 cases a motorized dental burr was used to create a $4.5 \mathrm{~cm}$ trough in the cortex of the humerus in the broad area of the avulsed pectoralis major insertion just lateral to the longhead of the biceps (Figure 4). In the first case of the series (Patient 1), the trough was created using a curette alone, however in the subsequent 5 cases it was discovered that the deployed military dentists had hand held motorized burrs that could be sterilized and used in the operating room. Thus in the successive cases the $4 \mathrm{~mm}$ motorized burr was borrowed from the dental clinic and utilized to create the trough. Next three $2.0 \mathrm{~mm}$ drill holes were placed in the humerus lateral to the trough. Free curved needles were then used to shuttle the \#2 Fiber wire suture through the trough out the drill holes and the tendon was tied down over an osseous bridge. Care was taken to make sure the tendon stump was seated completely into the trough. The wound was then irrigated, the repair inspected and hemostasis was obtained using electro-cautery. Indwelling drains were not utilized. 3-O braided suture was used to close the deltopectoral fascia. The wound was again irrigated and subcutaneous skin was closed using a 3-0 absorbable monofilament suture was placed in a buried interrupted fashion with overlying 3-0 nylon placed in a horizontal mattress fashion. All patients were

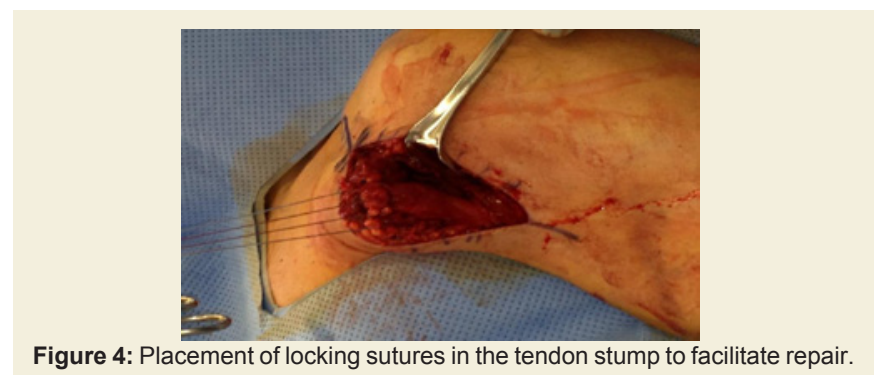

Figure 4: Placement of locking sutures in the tendon stump to facilitate repair.

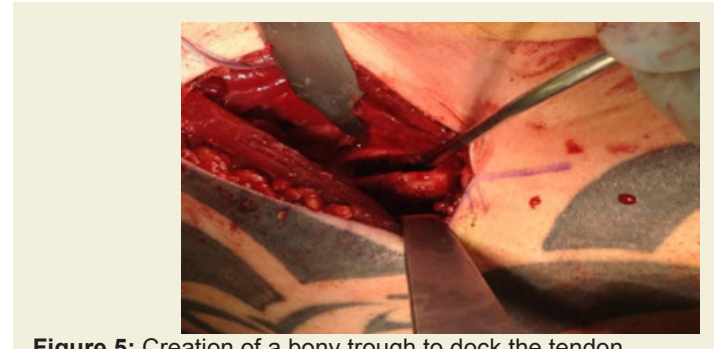

Figure 5: Creation of a bony trough to dock the tendon. 
Citation: Salazar D, Shakir I, Israel H, Choate WS, Joe K, et al. Acute Pectoralis Major Tears in Forward Deployed Active Duty U.S. Military Personnel: A Population at Risk?. J Orthopedics Rheumatol. 2018; 5(1): 6.

ISSN: $2334-2846$

Table 2: Patient Demographics.

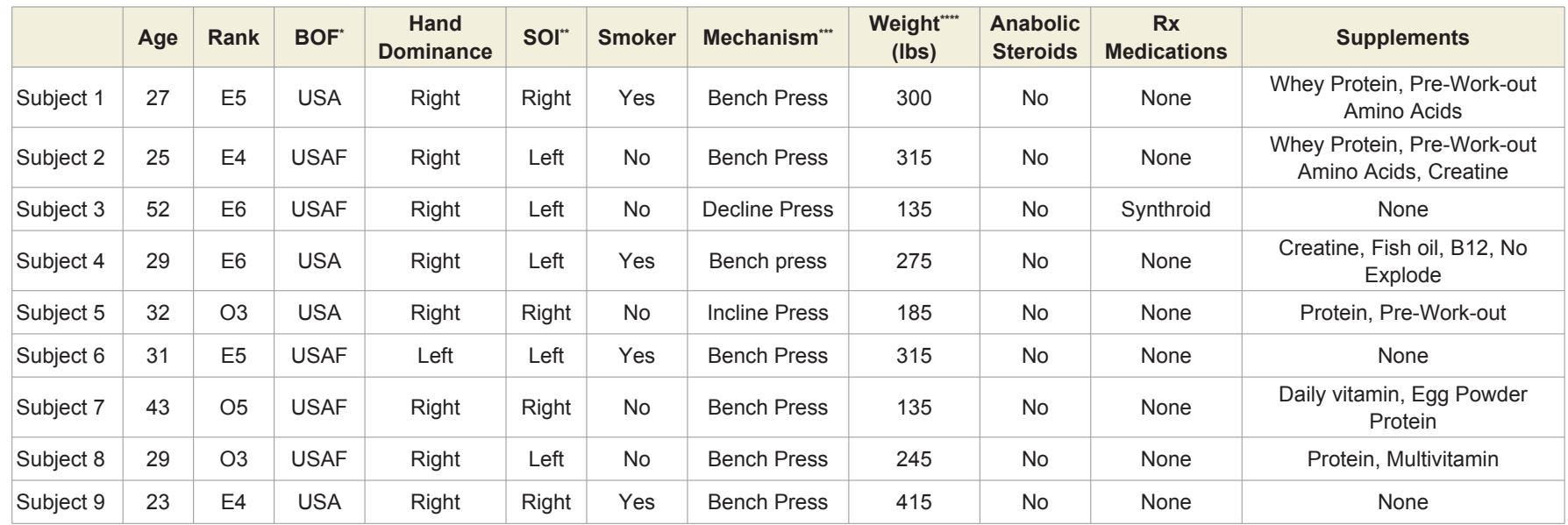

"BOF: Branch of Service; " SOI: Side of Injury; "** Mechanism: Mechanism of Injury; "*tweight: Weight on the Bench Press Bar at the Time of Injury

discharged with a sling for the operative extremity.

\section{Postoperative management \& rehabilitation}

All surgical patients underwent the same physical therapist supervised rehabilitation regiment. Patients were seen back for their first post-operative visit and wound check at 7 days and subsequently again at 14 days for suture removal. Post-operative restrictions were as follows:

Phase 1: (post-op to 6 weeks)-active and active assisted ROM of the elbow, wrist and hand. Pendulum/Codman's exercises of the shoulder were initiated. No lifting or weight bearing with the operative upper extremity. No active internal rotation or passive external rotation of the shoulder. No shoulder abduction.

Phase 2: (6 weeks to 4 months)-The sling was discontinued completely and patients began therapist supervised progressive active and active assisted range of motion of the shoulder. Terminal forced passive external rotation or abduction was prohibited. No lifting of objects greater than $5 \mathrm{lbs}$.

Phase 3: (4 months to 6 months)-Patients continued in therapy and were advanced to unlimited active, active assisted and passive range of motion of the left shoulder. Progressive light strengthening was initiated.

Phase 4: (6 months)-Patients were allowed unrestricted stretching and strengthening, and were allowed to resume full activity. Therapy was discontinued.

\section{Results}

The Titjen classification was utilized to classify pectoralis major tears in forward deployed active duty military personnel (Table 1). Nine male patients (5 US Air Force, 4 US Army) were diagnosed with pectoralis major tears; their demographics are presented in Table 2. Seven complete tears at the tendinous insertion (Tietjen Type III-D), 1 complete tear at the musculotendinous junction (Tietjen Type III-C) and 1 incomplete tear (Tietjen Type II) with a mean age of 32 years (23-52). All injuries occurred during bench press with a mean weight of $258 \mathrm{lbs}(135-415 \mathrm{lbs})$, with the dominant upper extremity involved $56 \%$ of the time. Table 3 demonstrates the mean length of deployment was 230 days (120-365), with injury occurring an average of 77 days into the tour (3-198 days). Mean time from injury to surgical repair in the cohort was 18 days (10-43). Table 4 details the MRI findings of the cohort.

\section{Discussion}

Rupture of the pectoralis major is a relatively rare occurrence with a recent systemic review of the literature demonstrating roughly only three hundred reported cases in the last 20 years [5]. The majority of cases in the modern literature have occurred in athletes, in particular weight lifters $[1-5,7,8]$. The pectoralis major is a powerful adductor and internal rotator of the arm. While low-demand patients may have no difficulty with activities of daily living after non-operative treatment, the inability to attain full strength without surgical repair necessitates operative treatment for the majority of athletes and laborers $[2,5,7,12]$.

In a recent prospective study performed on sixty consecutive patients with pectoralis major ruptures, the bench-press exercise during weight lifting was implicated in $80 \%$ of the cases [2]. In our all military cohort, bench press (6 flat bench, 1 incline, 1 decline) was the mechanism of injury in $100 \%$ of the cases. The injuries are theorized to have occurred when a maximal force was applied with the pectoralis major eccentrically contracting during an abducted and externally rotated shoulder position. Anatomic cadaveric studies have demonstrated that in the terminal 30 degree arc of shoulder extension, the shorter fibers of the inferior sternal head segments of the pectoralis major muscle lengthened $30 \%$ to $40 \%$ of their resting fiber length. This is nearly twice the amount of maximal excursion demonstrated in the more superior muscle fibers of the clavicular head

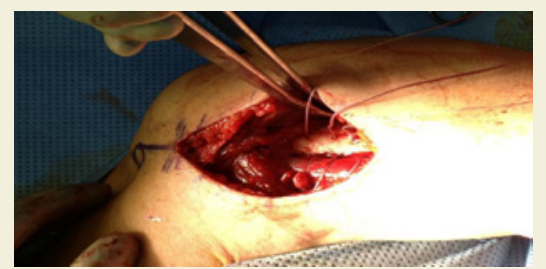

Figure 6: Trans-osseous repair with tendon docking into a through and sutures taken through lateral drill holes and tied over a bony bridge. 
Citation: Salazar D, Shakir I, Israel H, Choate WS, Joe K, et al. Acute Pectoralis Major Tears in Forward Deployed Active Duty U.S. Military Personnel: A Population at Risk?. J Orthopedics Rheumatol. 2018; 5(1): 6.

ISSN: $2334-2846$

Table 3: Deployment details, timing of injury and surgery.

\begin{tabular}{|l|c|c|c|c|c|c|c|c|}
\hline & Age & BOF & Rank** & $\begin{array}{c}\text { Deployment } \\
\text { Length (Days) }\end{array}$ & $\begin{array}{c}\text { Time Of } \\
\text { Injury*** } \\
\text { (Days) }\end{array}$ & $\begin{array}{c}\text { Tietjen } \\
\text { Type }\end{array}$ & MRI & Location of Surgery \\
\hline Subject 1 & 27 & USA & E5 & 365 & 120 & III-D & No & AUAB \\
\hline Subject 2 & 25 & USAF & E4 & 270 & 5 & III-D & Yes & AUAB \\
\hline Subject 3 & 52 & USAF & E6 & 120 & 30 & III-D & Yes & AUAB \\
\hline Subject 4 & 29 & USA & E6 & 270 & 103 & II & Yes & AUAB \\
\hline Subject 5 & 32 & USA & O3 & 270 & 3 & III-D & Yes & AUAB \\
\hline Subject 6 & 31 & USAF & E5 & 180 & 61 & III-C & Yes & N/A \\
\hline Subject 7 & 43 & USAF & O5 & 180 & 30 & III-D & Yes & N/A \\
\hline Subject 8 & 29 & USAF & O3 & 148 & 145 & III-D & No & N/A \\
\hline Subject 9 & 23 & USA & E4 & 270 & 198 & III-D & No & Nadigan \\
\hline
\end{tabular}

"BOF: Branch of Service; "*Rank: Military Rank; E: Enlisted; O: Officer

${ }^{* * *}$ Number of into the planned deployment that the injury occurred; ${ }^{* * * t} A U A B: 379^{\text {th }}$ Expeditionary Medical Group; Al Udeid Air Base; ${ }^{* * *+*}$ Madigan Army Medical Center Tacoma; WA

Table 4: Advanced imaging results and injury classification.

\section{Official MRI Report by Radiologist}

Subject 2 "Area of altered signal intensity seen at lateral portion of left pectoralis major muscle measured $4 X 2.5 X 1.9 \mathrm{~cm}$ in $3 \mathrm{orthogonal}$ planes, it appeared iso/ Subject 2 hypointwnaw suggestive of muscle tear with intramuscular hematoma surrounded by residual edema"

Subject 3 "Fluid signal intensity is seen intramuscular belly of left pectoralis major muscle with fluid filling, the defect in the muscle belly evidence of complete torn Subject 3 muscle fibers. Soft tissue edema is seen anterior to left pectoralis muscle."

Subject $4 \quad$ "MRI findings of left shoulder are consistent with acute complete tear of pectoralis major muscle, myotendinous junction. There is associated hemorrhagic fluid surrounding the torn retracted fibers"

Subject 5 "MRI findings are consistent with high-grade partial tear of the right pectoralis major at its musculotendinous junction, with surrounding edema and Subject 5 hemorrhage and subsequent mild retraction of its muscle fibers"

Subject $6 \quad$ "There is a complete tear of the musculotendenous junction of the sternal head of the pectoralis major muscle with gap/adjacent \%X4cm cystic like lesion Subject 6 with subtle layering suggestive of hematoma"

Subject 7 "There is a suspicion of partial tear of the pectoralis major tendon"

and the upper segments of the sternal head [6]. Thus, heavy weights applied during eccentric loading in the deep position of the bench press exercise put the inferior sternal head segments at an extreme mechanical disadvantage and susceptible to rupture. Our experience supports this theory; in the sole patient with an incomplete rupture, the clavicular head and a small portion of the superior segments of the sternal head were found intact while the remaining inferior sternal segments were avulsed from their humeral insertion.

Our series was comprised of 7 complete tears at the tendinous insertion (Tietjen Type III-D), 1 complete tear at the musculotendinous junction (Tietjen Type III-C) and 1 incomplete tear (Tietjen Type II), supporting previous reports that complete tears at the tendinous insertion are the most common type of pectoralis major rupture $[1,2,5,6,11]$. In the largest prospective study of pectoralis major injuries, the anatomic location of the tear was described to be at the humeral insertion of the tendon in 57 of 60 patients (95\%) [2]. When the location of the injury or the diagnosis itself is in doubt, magnetic resonance imaging (MRI) has been proposed in delineating the site and extent of the rupture, which may help surgeons with treatment options and surgical planning [13-16]. However due to the complex local anatomy and the rarity of the injury it can be difficult on routine MRI to differentiate separate areas of muscle and tendon at the distal myotendinous junction [13]. Previous authors have recommended obtaining images with the upper extremity in the abducted and externally rotated position, the so called "ABER" position to aid in differentiation [13]. Additionally the use of a surface coil is proposed to increases the signal-to-noise ratio, enhance spatial resolution and increase the conspicuity of the injury [14]. In our series, MRI imaging was obtained for the first six patients. Because MRI imaging was not available at the American military treatment facility, we had to rely on local foreign hospitals, which often involved substantial time delays, exorbitant costs and long distance travel. Table X details the MRI interpretations of the patients that underwent advance imaging. In our series the official interpretation of the images correlated poorly with both the intra-operative grade and anatomic location of the injury. Furthermore the images were found to be of lower quality and often difficult for the operating surgeon to interpret. Thus advanced imaging was abandoned, as the additional data was not found to impact our treatment plans. We conclude that routine MRI imaging in forward deployed locations is of limited value in the setting of acute pectoralis major tears.

While the operative goal of all surgical repair techniques remains suture restoration to achieve anatomic reduction of the tendon footprint while avoiding injury to the adjacent long head of the biceps, several methods have been described to reattach the avulsed tendon to its normal humeral insertion. These techniques have included suture anchors, cortical buttons and troughs with transosseous repair [1,2,5,7,12,17-20]. Biomechanical studies have sought to compare the strengths of the various techniques in an attempt to guide operative repair $[18,20,21]$. In a recent cadaveric load to failure study of pectoralis major tendon repairs in the extended and abducted position, Rabuck SJ et al. found that bone trough repair was stronger 
Citation: Salazar D, Shakir I, Israel H, Choate WS, Joe K, et al. Acute Pectoralis Major Tears in Forward Deployed Active Duty U.S. Military Personnel: A Population at Risk?. J Orthopedics Rheumatol. 2018; 5(1): 6.

ISSN: 2334-2846

than suture anchor repair [21]. In our case series of forward deployed active duty military personnel, all complete ruptures were surgically repaired using bone trough and transosseous repair. This repair technique was not chosen solely due to the current biomechanical findings, but rather also out of necessity. Due to the deployed operating room setting, orthopedic implants such as cortical buttons and suture anchors are unavailable. The mechanism to supply, stock and maintain such implants at forward military hospitals does not currently exist.

Due to the uncommon nature of this injury the literature is lacking of large prospective studies, which makes identification of statistically significant modifiable risk factors and high-risk populations difficult. However male gender has been nearly ubiquitous in the reported cases throughout history and clearly remains a risk factor [1-8,10$12,19,22]$. Additionally in a prospective study of 48 patients that sustained pectoralis major ruptures during bench press, $95.8 \%$ were found to have used anabolic steroids [2]. Our series included all men, but none admitted to anabolic steroid usage. Because steroid usage among active duty military personnel is punishable under the Uniformed Code of Military Justice, there is a disincentive for disclosure and thus candid answers cannot be assured in this patient population.

Although pectoralis major ruptures have previously been described in US military personnel 8-10, there has never been a description of an increased incidence among forward deployed troops. In a 4-month time period from December 2013 to March 2014 we saw acute pectoralis major ruptures in a forward deployed patient population with alarming frequency. We hypothesize that this can be attributed to the increase in both intensity and frequency of physical training that occurs during deployments to the war zone. We sought to identify a time period during deployment that subjects were more vulnerable to this injury, however no temporal relationship could be identified as a risk factor as we had one patient rupture 3 days into his deployment, while another patient ruptured 7 days prior to completion of his tour of duty.

Due to the population make-up and size of our study, there are several limitations. First, because of the rigorous physical demands of the armed forces and the unique environment of a forward deployed combat zone, our findings may not be generalizable to other patient populations. Additionally, due to the relatively small size of our cohort, identification of independent modifiable risk factors is not statistically possible. The constant cycle of forward deployment, geographic separation, operational tempo and redeployment to home station for both the military physicians and patients limits the ability to perform routine follow-up or obtain long term validated outcomes scores.

\section{Conclusion}

In Conclusion, due to the alarming frequency with which pectoralis major ruptures were diagnosed and treated at one expeditionary military treatment facility over a four-month deployment cycle, deployed active duty US military personnel likely represent a highrisk population for this injury. Future studies designed to identify the true incidence, report long-term outcomes and investigate modifiable patient risk factors in this population are warranted. Surgical repair with a trough and transosseous repair technique is possible in the forward deployed setting and may be considered on a case-by-case basis.

\section{References}

1. Bak K, Cameron EA, Henderson IJ (2000) Rupture of the pectoralis major: a meta-analysis of 112 cases. Knee Surg Sports Traumatol Arthrosc 8: 113119

2. de Castro Pochini A, Andreoli CV, Belangero PS, Figueiredo EA, Terra BB et al. (2014) Clinical considerations for the surgical treatment of pectoralis major muscle ruptures based on 60 cases: a prospective study and literature review. Am J Sports Med 42: 95-102.

3. Hanna CM, Glenny AB, Stanley SN, Caughey MA (2001) Pectoralis majo tears: comparison of surgical and conservative treatment. $\mathrm{Br} \mathrm{J}$ Sports Med 35: 202-206.

4. Schepsis AA, Grafe MW, Jones HP, Lemos MJ (2000) Rupture of the pectoralis major muscle. Outcome after repair of acute and chronic injuries. Am J Sports Med 28: 9-15.

5. EIMaraghy AW, Devereaux MW (2012) A systematic review and comprehensive classification of pectoralis major tears. J Shoulder Elbow Surg 21: 412-422.

6. Wolfe SW, Wickiewicz TL, Cavanaugh JT (1992) Ruptures of the pectoralis major muscle. An anatomic and clinical analysis. Am J Sports Med 20: $587-$ 593.

7. Petilon J, Carr DR, Sekiya JK, Unger DV (2005) Pectoralis major muscle injuries: evaluation and management. J Am Acad Orthop Surg 13: 59-68.

8. Zacchilli MA, Fowler JT, Owens BD (2013) Allograft reconstruction of chronic pectoralis major tendon ruptures. J Surg Orthop Adv 22: 95-102.

9. Hoppes CW, Ross MD, Moore JH (2013) Undetected pectoralis major tendon rupture in a patient referred to a physical therapist in a combat environment: a case report. Phys Ther 93: 1225-1233.

10. Shepard NP, Westrick RB, Owens BD, Johnson MR (2013) Bony avulsion injury of the pectoralis major in a 19 year-old male judo athlete: a case report. Int J Sports Phys Ther 8: 862-870.

11. Tietjen $R$ (1980) Closed injuries of the pectoralis major muscle. J Trauma 20: $262-264$

12. Aarimaa V, Rantanen J, Heikkila J, Helttula I, Orava S (2004) Rupture of the pectoralis major muscle. Am J Sports Med 32: 1256-1262.

13. Carrino JA, Chandnanni VP, Mitchell DB, Choi-Chinn K, DeBerardino TM, et al. (2000) Pectoralis major muscle and tendon tears: diagnosis and grading using magnetic resonance imaging. Skeletal Radiol 29: 305-313.

14. Connell DA, Potter HG, Sherman MF, Wickiewicz TL (1999) Injuries of the pectoralis major muscle: evaluation with MR imaging. Radiology 210: 785791.

15. Lee J, Brookenthal KR, Ramsey ML, Kneeland JB, Herzog R (2000) MR imaging assessment of the pectoralis major myotendinous unit: an MR imaging-anatomic correlative study with surgical correlation. AJR Am J Roentgenol 174: 1371-1375.

16. Ohashi K, El-Khoury GY, Albright JP, Tearse DS (1996) MRI of complete rupture of the pectoralis major muscle. Skeletal Radiol 25: 625-628.

17. Metzger PD, Bailey JR, Filler RD, Waltz RA, Provencher MT, et al. (2012) Pectoralis major muscle rupture repair: technique using unicortical buttons. Arthrosc Tech 1: e119-e125.

18. Hart ND, Lindsey DP, McAdams TR (2011) Pectoralis major tendon rupture: a biomechanical analysis of repair techniques. J Orthop Res 29: 1783-1787.

19. Lau BH, Butterwick DJ, Lafave MR, Mohtadi NG (2013) Retrospective review of pectoralis major ruptures in rodeo steer wrestlers. Adv Orthop 2013 987910.

20. Sherman SL, Lin EC, Verma NN, Mather RC, Gregory JM, et al. (2012) 
Citation: Salazar D, Shakir I, Israel H, Choate WS, Joe K, et al. Acute Pectoralis Major Tears in Forward Deployed Active Duty U.S. Military Personnel: A Population at Risk?. J Orthopedics Rheumatol. 2018; 5(1): 6.

\section{ISSN: 2334-2846}

Biomechanical analysis of the pectoralis major tendon and comparison of techniques for tendo-osseous repair. Am J Sports Med 40: 1887-1894.

21. Rabuck SJ, Lynch JL, Guo X, Zhang LQ, Edwards SL, et al. (2012) Biomechanical comparison of 3 methods to repair pectoralis major ruptures. Am J Sports Med 40: 1635-1640.
22. Wheat Hozack MJ, Bugg B, Lemay K, Reed J, Canadian Pro Rodeo Sport Medicine (2013) Tears of pectoralis major in steer wrestlers: a novel repair technique using the EndoButton. Clin J Sport Med 23: 80-82. 